\title{
Ocular toxocariasis in a patient with repeatedly negative ELISA titre to Toxocara canis
}

\author{
James A Sharkey, Peter S McKay
}

Infection caused by the larvae of Toxocara canis is a well established cause of intraocular inflammation. This frequently occurs in children and young adults. There are three recognised forms, diffuse panuveitis, a focal posterior retinal granuloma, or peripheral inflammatory mass. The peripheral inflammatory mass is often associated with vitreous bands, which may cause traction on the retina or optic disc. ${ }^{1-5}$

\section{Case report}

A 16-year-old farmer's son attended the opthalmic outpatient department. He gave a 1 month history of gradually reducing left visual acuity associated with the appearance of floaters.

On examination left visual acuity (VA) was reduced to $6 / 12$, right VA was recorded at $6 / 6$. Anterior segment examination revealed a moderate anterior uveitis $(2+$ cells). On posterior segment examination, there was a marked vitritis which somewhat obscured fundal details; however a pale mass was noted in the retinal periphery in the 7 o'clock meridian.

The patient was admitted for the investigation of panuveitis. A number of laboratory tests were carried out. Full blood count showed a slight leucocytosis of $12 \cdot 3 \times 10^{9} /$. Differential white cell count showed an eosinophilia of $5 \%$. The Toxoplasma enzyme-linked immunosorbent assay (ELISA) test was IgM and IgG negative. The serum Toxocara ELISA test was negative at a 1:4 dilution (optical density (OD) 0.07). At this time only the anterior uveitis was treated.

Three weeks later the left VA had dramatically reduced to counting fingers (CF). Examination

Figure 1 Peripheral lesion typical of Toxocara

granuloma. showed a marked vitritis with associated cystoid macular oedema. Inferonasally a pale mass was again visible having many of the characteristics of a peripheral Toxocara granuloma (Fig 1). There was also a vitreous traction band extending from this mass to the optic disc. A clinical diagnosis of peripheral Toxocara granuloma with associated endophthalmitis was made, and the patient was treated with topical, oral, and peribulbar steroids. The serum Toxocara ELISA test was repeated but again was negative at a 1:4 dilution (OD 0.08).

Following the steroid treatment left vision improved to $6 / 60$. However significant traction was being exerted by condensation of vitreous bands extending between the peripheral mass and the posterior pole structures. Pars plana vitrectomy was undertaken 1 week later and segmentation of these bands performed. A sample of vitreous obtained at this time was submitted for a Toxocara ELISA test. The vitreous titres were greatly elevated at a 1:8 dilution (OD 0.51). Postoperatively vision improved gradually, and 9 months later the left vision is $6 / 12$.

\section{Comment}

The diagnosis of ocular toxocariasis has been improved with the introduction of the ELISA test. This is positive in $90 \%$ of patients suspected of having clinical Toxocara infection. ${ }^{6}$ However laboratory reporting of a negative serum ELISA result may lead to error in diagnosis. Pollard and colleagues ${ }^{6}$ have suggested that there is significant local antibody production in ocular toxocariasis, demonstrating that serum $T$ canis antibody is on average four times lower in patients with ocular involvement when compared with patients with systemic visceral larvae migrans. Biglan and co-workers ${ }^{7}$ have also demonstrated ELISA titres in vitreous specimens to be substantially higher than in serum in ocular toxocariasis. Similar findings have been demonstrated in the aqueous humour of infected patients. ${ }^{8}$ An eosinophilia of the aqueous has also been demonstrated in ocular toxocariasis. ${ }^{9}$

Clinicians should be aware that the serum ELISA with ocular nematode infection by $T$ canis may be low $^{1011}$ or, as in this case, negative. However the test may be strongly positive and therefore of great diagnostic importance if carried out on intraocular fluid from an infected patient. This will be especially important in childhood where a clinically similar picture may be caused by retinoblastoma." Sampling may take the form of aqueous tap or vitreous biopsy, if therapeutic pars plana vitrectomy is performed. 
1 Wilder HC. Nematode endophthalmitis. Trans Am Acad Ophthalmol Otolaryngol 1950; 55: 99-109.

Duguid IM. Chronic endophthalmitis due to Toxocara Brf Ophthalmol 1961; 45: 705-17.

3 Duguid IM. Features of ocular infestation by Toxocara. Brf Ophthalmol 1961; 45: 789-96.

4 Asthon N. Larval granulomatosis of the retina due to Toxocara. Brf Ophthalmol 1960; 44: 129-48.

5 Wilkinson CP, Welch RB. Intraocular Toxocara. Am $\mathcal{f}$ Ophthalmol 1971; 71: 921-30.

6 Pollard ZF, Jarrett WH, Hagler WS, Allain DS, Schantz PM. ELISA for diagnosis of ocular toxocariasis. Ophthalmology 1979; 86: 743-9.
7 Biglan AW, Glickman LT, Lobes LA. Serum and vitreous Toxocara antibody in nematode endophthalmitis. Am $\mathcal{J}$ Ophthalmol 1979; 88: 898-901.

8 Felberg NT, Shields JA, Federman JL. Antibody to Toxocara canis in the aqueous humor. Arch Ophthalmol 1981; 99: $1563-4$.

9 Shields JA, Lerner HA, Felberg NT. Aqueous cytology and enzymes in nematode endophthalmitis. Am $\mathcal{F}$ Ophthalmo enzymes in nemat

10 Kielar RA. Toxocara canis endophthalmitis with low ELISA titre. Ann Ophthalmol 1983; 15: 447-9.

11 Searl SS, Moazed K, Albert DM, Marcus LC. Ocular toxocariasis presenting as leukocoria in a patient with low ELISA titre to Toxocara canis. Ophthalmology 1981; 88: 1302-6.

\section{The trap of the displaced blind spot in automated perimetry}

Neuro-ophthalmology Unit, Geneva University Hospital, Geneva,

Switzerland

A B Safran

G Sunaric

Division of

Endocrinology, Geneva

University Hospital

Geneva, Switzerland

A Burger

Correspondence to:

Professor Avinoam B Safran, Neuro-ophthalmology Unit, Geneva University Hospital, 1211 Geneva 4, Switzerland.

Accepted for publication

6 January 1993

\section{Avinoam B Safran, Gordana Sunaric, Albert Burger}

In routine computer programs used with automated perimetry, restriction in the number of tested locations results in poor spatial delineation of the blind spot. To avoid an inadequate interpretation of the recorded data, a number of computer programs ${ }^{1}$ exclude the evaluation of points located within the presumed area of the blind spot and its surroundings. These programs leave an empty space in the corresponding area of the result printouts.

This solution is advantageous in most clinical settings. With marked cyclotorsion, however,

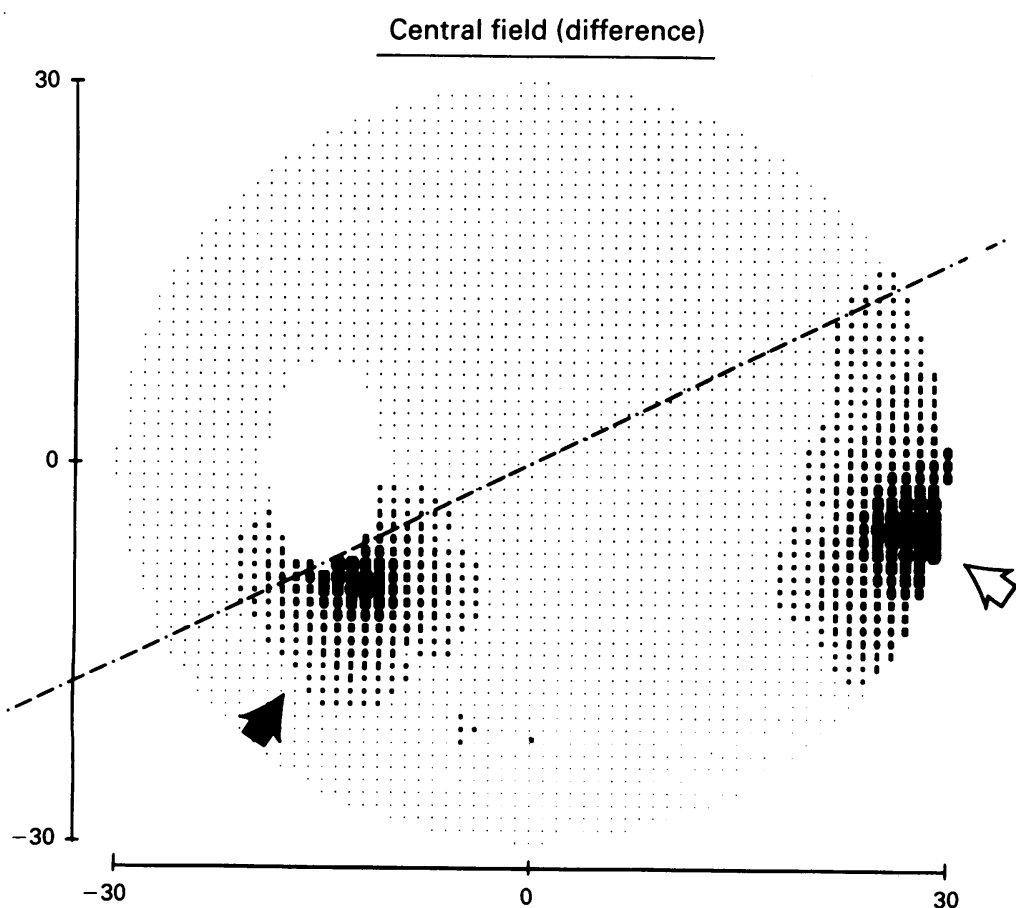

Figure 1 Left visual field, evaluated using automated perimetry. It demonstrated an inferonasal defect (open arrow), and a displaced, enlarged blind spot (closed arrow), and this is mimicking a profoundly altered area within the bundle defect. Broken line shows the projection of the papillomacular axis into the visual field, as inferred from fundus evaluation. the blind spot is displaced to such an extent that it appears in the evaluated portion of the visual field. The condition may be confusing when it occurs in conjunction with a bundle defect, mimicking an absolute scotoma within the area of the nerve fibre defect. This was shown with a patient suffering from Graves' disease which resulted in severe excyclotorsion and optic neuropathy in the same eye.

\section{Case report}

A 57-year-old woman presented with endocrine orbitopathy. Corrected visual acuity was 20/20 in the right eye and 20/25 in the left eye. Relative afferent pupillary defect was found in the left eye. With the patient's head carefully placed in a straight position against the rests, Octopus automated perimetry was performed using the $30^{\circ}$ option of the $\mathrm{N} 1$ program ${ }^{2}$ (Interzeag AG, Schlieren, Switzerland). In the right eye, visual field was unremarkable. In contrast, in the lef eye, the examination showed nasal defects, and a dense scotoma at the inferonasal border of the unchecked area of the blind spot (Fig 1). In that eye, Goldmann kinetic perimetry showed an inferior nasal step; in addition, the blind spot was located some $20^{\circ}$ below its usual position, indicating that the absolute scotoma observed with automated perimetry represented a displaced blind spot. Left fundus showed a $25^{\circ}$ excyclotorsion (Fig 2). Ocular motility was impaired in both eyes, with reduction in elevation and abduction. Exophthalmometry was recorded as 14-14/107. Ocular pressure was markedly increased when attempting upgaze.

\section{Comment}

Displacement of the blind spot has been described in eyes with cyclotorsion. ${ }^{3}$ However, no emphasis has been given to the confounding 